\title{
Oral Immunization of FMDV Vaccine Using pH-Sensitive and Mucoadhesive Thiolated Cellulose Acetate Phthalate Microparticles
}

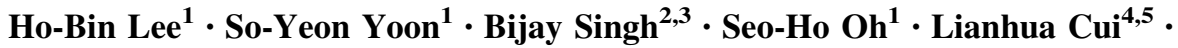 \\ Changguo Yan $^{4,5} \cdot$ Sang-Kee Kang ${ }^{6}$ Yun-Jaie Choi ${ }^{1,2} \cdot$ Chong-Su Cho ${ }^{1,2}$
}

Received: 21 July 2017/Revised: 28 August 2017/ Accepted: 4 September 2017/Published online: 17 October 2017

(C) The Korean Tissue Engineering and Regenerative Medicine Society and Springer Science+Business Media B.V. 2017

\begin{abstract}
Several barriers such as gastric pH, enzymatic degradation and rapid transit should be overcome to orally deliver antigens for taking up by epithelial microfold cells in Peyer's patches of small intestine. To solve the above mentioned problems, we designed $\mathrm{pH}$-sensitive and mucoadhesive polymeric microparticles (MPs) prepared by double emulsion technique using cellulose acetate phthalate (CAP) to enhance immune response of foot-and-mouth disease (FMD) virus (FMDV) subunit vaccine. Thiolation of CAP improved mucoadhesive property of CAP to prolong the MPs transit time through the gastrointestinal tract. Thiolated CAP (T-CAP) also slowed down antigen release in acidic $\mathrm{pH}$ of stomach but released more antigens in neutral $\mathrm{pH}$ of small intestine due to the $\mathrm{pH}$-sensitivity of the T-CAP. Oral immunization of a chimerical multi-epitope recombinant protein as the FMD subunit vaccine via T-CAP MPs effectively delivered the vaccine to Peyer's patches eliciting mucosal IgA response. It will make a step forward into a promising oral subunit vaccine development in livestock industry.
\end{abstract}

Keywords pH-senstive $\cdot$ Mucoadhesive $\cdot$ Foot-and-mouth disease $\cdot$ Subunit vaccine $\cdot$ Oral immunization

Ho-Bin Lee and So-Yeon Yoon have contributed equally to this work.

Yun-Jaie Choi

cyjcow@snu.ac.kr

$\triangle$ Chong-Su Cho

chocs@snu.ac.kr

1 Department of Agricultural Biotechnology, Seoul National University, 1 Gwanak-ro, Gwanak-gu, Seoul 08826, Republic of Korea

2 Research Institute for Agriculture and Life Science, Seoul National University, 1 Gwanak-ro, Gwanak-gu, Seoul 08826, Republic of Korea

3 Research Institute for Bioscience and Biotechnology, Kathmandu 44600, Nepal

4 Department of Animal Science, Yanbian University, Yanji 133002, Jilin, China

5 Co-Innovation Center of Beef Cattle Science and Industry Technology, Yanbian University, Yanji 133002, Jilin, China

6 Institute of Green-Bio Science and Technology, Seoul National University, 1447-1 Pyeongchang-Daero, DaehwaMyeon, Pyengchang-Gun, Gangwon-Do 25354, Republic of Korea

\section{Introduction}

Foot and mouth disease (FMD) is a very high contagious virus disease occurred to cloven-hoofed animals such as sheep, goat, pig and cattle. Its outbreak has been affecting livestock industry because it is very difficult to cure the disease due to the continuous evolving and mutating of the virus [1]. To prevent infection of FMD, inactivated virus vaccines as traditional vaccines have been commercialized [2]. However, it is expensive because the production of inactivated vaccine requires a high level of biological safety facility to prevent risk of the spread of live virus during vaccine production, and has a serious biosafety concern, which would hinder efforts to employ serology to distinguish between infected and vaccinated animals (DIVA) [3]. To overcome the limitations of inactivated vaccine, subunit vaccines have been recently attracted because they can be produced in $E$. coli by relatively easy recombinant technique with a high production yield and a cost-efficiency without safety problems $[2,4]$. 
In our previous study, a chimerical multi-epitope recombinant protein, $\mathrm{M} 5 \mathrm{BT}$, composed of tandem repeats of five B cell epitopes derived from different FMD virus (FMDV) variants and one T-cell epitope as the subunit vaccine was successfully prepared and FMDV specific antibodies were successfully obtained in the serum of mice immunized with the designed M5BT [5].

Vaccination is aimed at generating immune response and memory response by innate and adaptive immune system of animals. Oral vaccine among mucosal immunization have been interested in the development of effective vaccine system because it has several advantages over parenteral vaccination, such as needle-free delivery, easy and comfortable administration [6]. Most importantly, oral vaccination can induce both mucosal and systemic immunity whereas parenteral immunization primarily induces a systemic immune responses [7, 8]. It is important to induce mucosal immune response because FMD is infected through the mucosa [9]. However, degradation of antigen in acidic stomach and enzymatic condition, low oral bioavailability and short residence time of antigen at target site make oral vaccination very difficult [10]. Therefore, orally administered vaccine requires protection from the harsh gastric condition and long residence time of vaccine at mucosal site $[8,11,12]$. Bifunctional polymers having $\mathrm{pH}$-sensitivity and mucoadhesivity have been used as effective carriers for oral vaccination.

Cellulose acetate phthalate (CAP) is an anionic polymer composed of phthalic acid and acetate. It shows $\mathrm{pH}$-sensitivity and is water-soluble only above pH 6.2 [10]. Hence, this can facilitate the efficient vaccine delivery to the gutassociated lymphoid tissue (GALT) located in the lower part of small intestine [13-15]. Thiolated polymers have been intensively used as mucoadhesive polymers because these polymers can form covalent bond between thiol groups of thiolated polymer and cysteine rich subdomain of mucus glycoproteins through disulfide bonds $[16,17]$. It is important to develop vaccine delivery carrier to prevent disease infected through mucosa. In this study, we developed pH-sensitive and mucoadhesive thiolated CAP (T$\mathrm{CAP}$ ) as a polymeric carrier. M5BT protein as a subunit vaccine for the FMD was encapsulated into T-CAP microparticles (MPs) using double emulsion solvent evaporation methods and the M5BT-loaded T-CAP MPs were characterized to investigate their efficacy in vitro and in vivo. As results, T-CAP MPs showed characteristics of $\mathrm{pH}$-sensitive and mucoadhesive properties, and improved the immune response of M5BT antigen.

\section{Materials and methods}

\subsection{Materials}

Cellulose acetate phthalate (CAP) was kindly provided from Shin-Etsu Chemical Co., Ltd. (Tokyo, Japan). N,N'dicyclohexylcarbodiimide (DCC), $N$-hydroxysuccinimide (NHS), L-cysteine hydrochloride monohydrate, dimethyl sulfoxide (DMSO), poly(vinyl alcohol) (PVA), Pluronic ${ }^{\circledR}$ F-127, dichloromethane (DCM), 5,5'-dithio-bis-(2-nitrobenzoic acid) (DTNB) and fluorescein diacetate (FDA) were purchased from Sigma-Aldrich (St. Louis, MO, USA).

Tris-glycine-PAG pre-cast SDS gel was provided by Komabiotech (Seoul, Korea). Bicinchoninic acid (BCA) protein assay reagents (A and B) were purchased from Thermo Scientific Pierce (Illinois, USA). Horseradish peroxidase (HRP)-conjugated goat anti-mouse IgA and IgG antibody were purchased from Santa Cruz Biotechnology (Dallas, TX, USA). Ellman's reagent was purchased from Novagen (California, USA).

\subsection{Preparation of T-CAP polymer}

\subsubsection{Synthesis of T-CAP}

The synthesis of T-CAP was carried out according to the method described previously [18]. Briefly, $4 \mathrm{~g}$ of CAP was dissolved in $100 \mathrm{ml}$ of DMSO and the carboxylic acid moieties of the polymer were activated by DCC (4.189 g) and NHS (2.337 g) with constant stirring at room temperature for $24 \mathrm{~h}$ under nitrogenous condition to avoid the oxidation of sulfhydryl groups by atmospheric oxygen. Byproducts were removed by filtration and the filtrate was further reacted with L-cysteine hydrochloride monohydrate $(0.355 \mathrm{~g})$ for $18 \mathrm{~h}$ under similar condition. The reaction mixture was filtered to remove by-products and the filtrate was dialyzed initially against $3 \mathrm{~L}$ of DMSO to remove the unbound L-cysteine hydrochloride and then against distilled water several times to remove DMSO. Finally, the polymer solution was lyophilized and the product was stored at $-20{ }^{\circ} \mathrm{C}$ until use. The conjugation of L-cysteine was confirmed by $600 \mathrm{MHz}{ }^{1} \mathrm{H}$ NMR spectroscopy (AVANCE 600, Bruker, Germany).

\subsubsection{Quantification of thiol group content in T-CAP}

The degree of thiol group substitution in the T-CAP was determined by Ellman's method according to the manufacturer's instructions. Briefly, $10 \mathrm{mg} / \mathrm{ml}$ of T-CAP solution was prepared and diluted with $0.1 \mathrm{M}$ sodium phosphate buffer $(\mathrm{pH}$ 8) containing $1 \mathrm{mM}$ EDTA to 
prepare different dilutions. $50 \mu \mathrm{l}$ aliquots of each dilution were added to $500 \mu \mathrm{l}$ of $0.5 \mathrm{M}$ phosphate buffer ( $\mathrm{pH} 8.0$ ) and $10 \mu \mathrm{l}$ of Ellman's reagent $(0.4 \mathrm{mg} / \mathrm{ml}$ of DTNB in $0.5 \mathrm{~mol} / \mathrm{l}$ phosphate buffer, $\mathrm{pH}$ 8.0). Control reactions were performed by with non-modified CAP. The samples were shielded from light and incubated at room temperatures for $15 \mathrm{~min}$. And then, $100 \mu \mathrm{l}$ of the supernatant was transferred to a micro titration plate and the absorbance was measured at $412 \mathrm{~nm}$ using microplate reader (TECAN Infinite 200 PRO). The amount of thiol groups was calculated from the standard curve prepared by measuring the absorbance of L-cysteine hydrochloride monohydrate solution as described above.

\subsection{Preparation of T-CAP MPs}

\subsubsection{Preparation of M5BT subunit vaccine}

The preparation of M5BT subunit vaccine for the FMDV was performed by the previously reported method [5].

\subsubsection{Preparation of M5BT-loaded T-CAP MPs}

M5BT-loaded T-CAP MPs were prepared using a water-inoil-in-water $\left(\mathrm{W}_{1} / \mathrm{O} / \mathrm{W}_{2}\right)$ double emulsion solvent evaporation method described previously with a little modification as shown in Fig. 1 [19]. $200 \mu \mathrm{l}$ of aqueous solution of M5BT (5 mg) was stabilized with $100 \mu \mathrm{l}$ of $10 \%$ Pluronic F-127 solution to form an internal aqueous phase $\left(\mathrm{W}_{1}\right)$. $100 \mathrm{mg}$ of each T-CAP and CAP was dissolved in $5 \mathrm{ml}$ of DCM and ethyl acetate/ethanol mixture $(1 / 1 \mathrm{v} / \mathrm{v})$, respectively. Organic phase was emulsified with the aqueous phase using an ultrasonic processor (Sonics, Vibra cells ${ }^{\mathrm{TM}}$ )
(4 output watts) on ice for $1.5 \mathrm{~min}$ to form $1^{\text {st }} \mathrm{W}_{1} / \mathrm{O}$ emulsion. The mixture emulsion was added drop by drop into $50 \mathrm{ml}$ of $1 \%(\mathrm{w} / \mathrm{v})$ PVA solution and then homogenized with Ultra Turrax (T25, IKA, Germany) at $13,000 \mathrm{rpm}$ for $1.5 \mathrm{~min}$ to form $\mathrm{W}_{1} / \mathrm{O} / \mathrm{W}_{2}$ emulsion. The resulting double emulsion was stirred for $4 \mathrm{~h}$ at room temperature to evaporate the organic solvent. After solvent evaporation, the hardened MPs were collected by centrifugation at $6000 \mathrm{rpm}$ for $10 \mathrm{~min}$, washed with distilled water, and lyophilized under vacuum. M5BT-loaded T-CAP and CAP MPs were stored at $-20^{\circ} \mathrm{C}$ until use. M5BT- loaded CAP MPs were prepared as similar with T-CAP MPs.

\subsubsection{Preparation of FDA-loaded T-CAP MPs (FDA/T- CAP MPs)}

FDA-loaded T-CAP and CAP MPs were similarly prepared as above-mentioned M5BT-loaded T-CAP MPs. $5 \mathrm{mg}$ of FDA was dissolved into $200 \mu \mathrm{l}$ of DCM, then added to T-CAP $(100 \mathrm{mg})$ solution dissolved in $5 \mathrm{ml}$ of DCM, and homogenized with $50 \mathrm{ml}$ of $1 \%$ (w/v) PVA solution using Ultra Turrax (T25, IKA, Germany) at 13,000 rpm for $1.5 \mathrm{~min}$ to form $\mathrm{O} / \mathrm{W}$ emulsion.

\subsection{Characterization of T-CAP MPs}

\subsubsection{Morphology by FE-SEM}

The surface topography was observed by field-emission scanning electron microscope (FE-SEM) using SUPRA 55VP-SEM (Carl Zeiss, Oberkochen, Germany). MPs were mounted on metal stubs with thin adhesive copper tape and

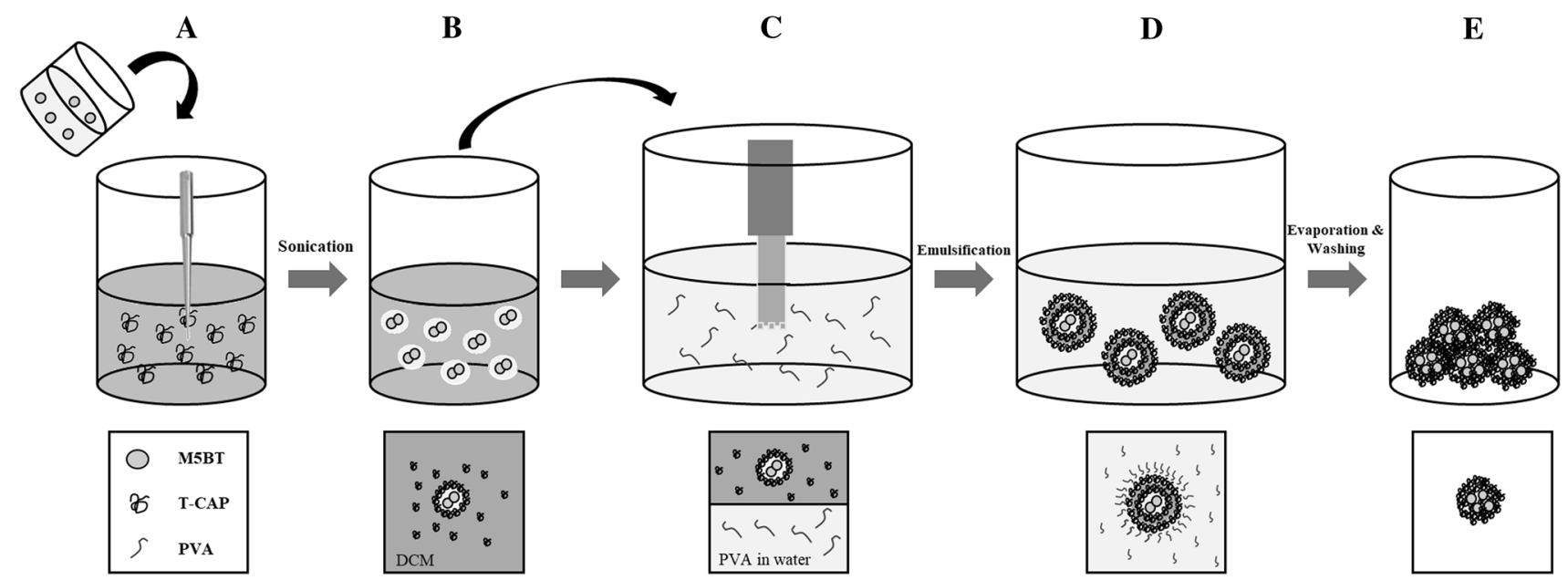

Fig. 1 Procedures of preparation for M5BT/T-CAP microspheres by double emulsion (modified from the figure from sander, soft matter, 2014). A Addition of M5BT solution (water phase, W1) into T-CAP solution in DCM (organic phase, O). B 1st emulsification (W1/O emulsion). C Addition of W1/O solution into PVA solution (water phase, W2). D 2nd emulsification (W1/O/W2 emulsion). E Hardened microsphere by evaporating DCM and centifugation 
coated with platinum under vacuum using coating chamber (CT $1500 \mathrm{HF}$, Oxford Instruments Oxfordshire, UK).

\subsubsection{Size by dynamic light scattering (DLS)}

The sizes of MPs were measured with a dynamic light scattering spectrophotometer (DLS-7000, Otsuka Electronics, Japan).

\subsubsection{Determination of loading content and loading efficiency}

Loading content was determined as follows. The MPs $(5 \mathrm{mg}$ ) were dispersed into $0.5 \mathrm{ml}$ of $0.1 \mathrm{M} \mathrm{NaOH}$ containing $0.5 \%(\mathrm{w} / \mathrm{v})$ SDS. The suspension was incubated in a water bath at $60{ }^{\circ} \mathrm{C}$ for $2 \mathrm{~h}$. Following centrifugation at $14,000 \mathrm{rpm}$ for $5 \mathrm{~min}, 0.5 \mathrm{ml}$ of the supernatant was withdrawn for BCA assay. The encapsulation efficiency of the M5BT into MPs was determined by measuring the unloaded protein concentration in the supernatant during the double emulsion method steps. The loading content and encapsulation efficiency of vaccine were calculated using the following equations:

$\begin{aligned} \text { Loading content }(\text { wt. }-\%)= & \frac{\text { amount of vaccine in MPs }}{\text { amount of MPs }} \\ & \times 100\end{aligned}$

Encapsulation efficienccy (wt. - \%)

$=\frac{\text { amount of vaccine in MPs }}{\text { amount of vaccine initially used }} \times 100$.

\subsubsection{In vitro release of vaccines from vaccine-loaded MPs}

In vitro release of M5BT from M5BT/CAP or M5BT/TCAP MPs was performed as follows. The MPs were placed into $1.5 \mathrm{ml}$ tubes with $0.5 \mathrm{ml}$ of $0.2 \mathrm{M}$ sodium phosphate buffer ( $\mathrm{pH} 7.4$ ) or $0.2 \mathrm{M} \mathrm{HCl}-\mathrm{KCl}$ buffer $(\mathrm{pH} 2)$ for $24 \mathrm{~h}$ at $37{ }^{\circ} \mathrm{C}$ with $100 \mathrm{rpm}$ shaking. A $0.5 \mathrm{ml}$ aliquot was withdrawn and replaced with an equal volume of each buffer at a predetermined time, and the amount of M5BT released was measured using spectrophotometer (NanoPhotomter ${ }^{\mathrm{TM}}$ ).

\subsubsection{Structural integrity of the M5BT released from MPs}

The structural integrity of M5BT before and after encapsulation in MPs was assessed by SDS-PAGE.

\subsubsection{Mucoadhesive property of MPs ex vivo}

Mucoadhesive property of MPs was evaluated using porcine intestinal mucosa. $4 \mathrm{mg}$ of each of FDA-loaded T-CAP MPs and FDA-loaded CAP MPs were dispersed on a freshly excised porcine intestinal mucosa which was fixed on a microscopic slide, and incubated at $37{ }^{\circ} \mathrm{C}$ for $1 \mathrm{~h}$ with shaking at $100 \mathrm{rpm}$. The MPs attached on the mucosa were collected, and remaining concentration of FDA at each MPs was calculated by interpolation from a standard curve measuring the absorbance of FDA at $490 \mathrm{~nm}$ $(\mathrm{n}=3)$.

\subsection{In vivo immunization in murine model}

\subsubsection{Animals and schedule of in vivo immunization}

5 female BALB/c mice of 7 weeks of age were used per group in this study. Mice were purchased from Samtako, Co. Ltd. (Osan, Korea) and housed in cages by providing ad libitum access to feed and water in accordance with the guidelines for the care and use of laboratory animals (Seoul National University). All of the protocols were reviewed and approved by the Animal Care and Use Committee at Seoul National University (SNU-150514-3-2). After 1 week of acclimatization, mice were orally immunized by oral gavage of MPs equivalent to $200 \mu \mathrm{g}$ of M5BT suspended in $200 \mu \mathrm{l}$ of PBS via a $1 \mathrm{ml}$ syringe fitted with an oral zonde for mouse $(20 \mathrm{G}, 5 \mathrm{~cm})$. All immunization groups received a total of 6 doses of vaccines such as 2 priming(day 0 , and 1 ) and 4 boosting (day $7,8,14$, and 15).

\subsubsection{Blood and fecal sampling}

Blood samples of immunized mice were collected into serum separating tube (BD vacutainer, UK) from tail vein at three points; before immunization, 2 weeks after primary immunization and 2 weeks after the last boosting immunization. The serum was separated from blood by centrifugation at $14,000 \mathrm{rpm}$ for $3 \mathrm{~min}$ and stored in $-20{ }^{\circ} \mathrm{C}$, and used for detection of antigen-specific antibodies by ELISA. Similarity, the feces of immunized mice were collected at the same time points of blood samples. Fecal pellets were homogenized in 10 volumes of resuspension buffer (PBS containing $1 \mathrm{mM} \mathrm{PMSF}$ and $1 \%$ BSA) at $4{ }^{\circ} \mathrm{C}$ overnight, centrifuged at $14,000 \mathrm{rpm}$ for $10 \mathrm{~min}$, supernatants were collected and analyzed for the presence of antigen-specific IgA by ELISA.

\subsubsection{Anti-M5BT antibody detection by ELISA}

Levels of serum M5BT-specific immunoglobulin G (total $\operatorname{IgG}$ ) and levels of $\operatorname{IgA}$ in the fecal samples with specificity to M5BT were determined by ELISA. Briefly, M5BT protein antigen $(1 \mu \mathrm{g} / \mathrm{ml})$ was diluted in carbonate buffer (pH 9.6) and diluted antigen was used for coating wells (100 $\mu \mathrm{l} /$ well) of 96-well immunoplate (SPL 32096). The plates were incubated at $37{ }^{\circ} \mathrm{C}$ for $2 \mathrm{~h}$ and washed with PBS $(200 \mu \mathrm{l} /$ well) for three times and blocked with block 


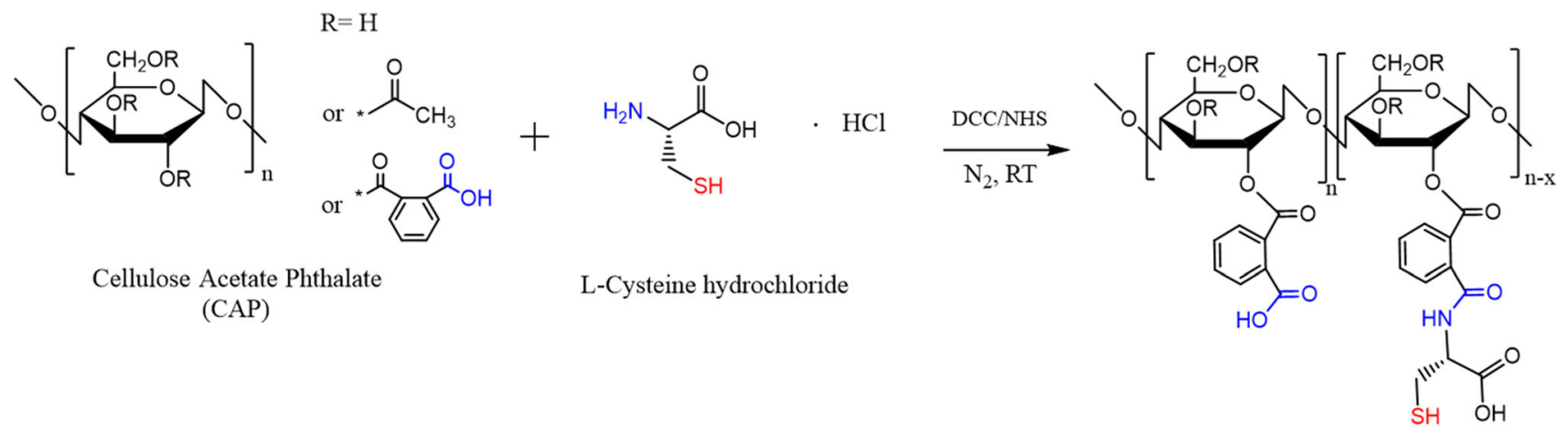

Thiolated CAP

Fig. 2 Synthesis scheme of thiolate Cellulose Acetate Phthalate (CAP)

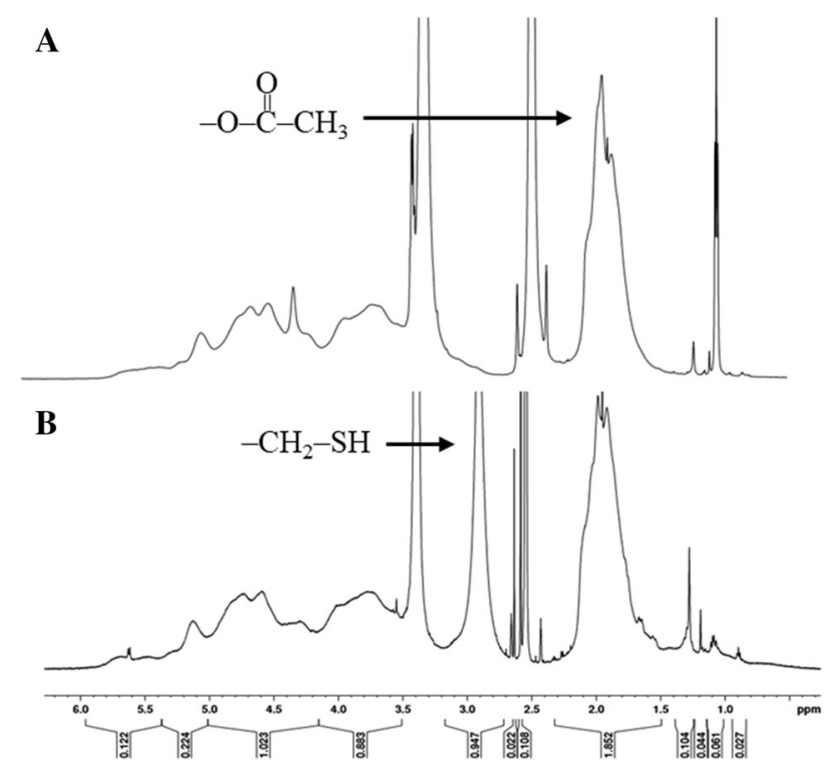

Fig. $3{ }^{1} \mathrm{H} 600 \mathrm{MHz}$ NMR spectra of A CAP and B T-CAP

buffer (PBS containing 1\% BSA) $(200 \mu \mathrm{l} /$ well) at room temperature for $1 \mathrm{~h}$. Following blocking at room temperature, mouse sera with a 1:100 dilution in blocking buffer were added to the wells (100 $\mu \mathrm{l} /$ well). For fecal samples, 1:25 diluent were used. Plates were incubated at $37{ }^{\circ} \mathrm{C}$ for $2 \mathrm{~h}$ followed by washing three times with PBST (PBS containing $0.05 \%$ Tween 20, $200 \mu \mathrm{l} /$ well). For specific antibodies detection, plates were incubated for $1 \mathrm{~h}$ at room temperature with appropriately diluted HRP-labeled goat anti-mouse immunoglobulin conjugates specific for IgG (1:5000 dilutions) or IgA (1:5000 dilutions). The plates were washed three times with PBST and then treated with TMB substrate solution (100 $\mu \mathrm{l} /$ well) for $5 \mathrm{~min}$ in the dark followed by the addition of stop solution $\left(0.16 \mathrm{M} \mathrm{H}_{2} \mathrm{SO}_{4}\right.$; $100 \mu \mathrm{l} /$ well) to stop the enzymatic reaction. Finally, the absorbance was measured at $450 \mathrm{~nm}$ using microplate reader (TECAN Infinite $200 \mathrm{PRO}$ ).
Table 1 Loading content and encapsulation efficiency of M5BTloaded MPs

\begin{tabular}{lll}
\hline Microparticles (MPs) & $\begin{array}{l}\text { Loading content } \\
\text { (wt. }-\%)\end{array}$ & $\begin{array}{l}\text { Encapsulation } \\
\text { efficiency (wt. -\%) }\end{array}$ \\
\hline M5BT/CAP MPs & $4.62 \pm 0.239$ & $82.2 \pm 3.27$ \\
M5BT/T-CAP MPs & $4.97 \pm 0.219$ & $72.1 \pm 1.36$ \\
\hline
\end{tabular}

\subsubsection{Flow cytometric detection of MHC class II- expressing cells in Peyer's patches}

After final sampling from the immunized mice, the mice were dissected to collect Peyer's patches from the ileum. Immune cells were further isolated as described earlier [20]. Briefly, a short ileum fragment with Peyer's patch was cut longitudinally and incubated at $37^{\circ} \mathrm{C}$ in $2 \mathrm{mM}$ EDTA in CMF HBSS buffer for three sequential $15 \mathrm{~min}$ incubations to remove the epithelial layer. Tissues were digested with $1.5 \mathrm{mg} / \mathrm{ml}$ Type VIII collagenase in CMF HBSS/FBS, and the resulting suspension of cells was passed through a 100 um cell strainer before centrifugation at $1500 \mathrm{rpm}$ for $5 \mathrm{~min}$ at $4{ }^{\circ} \mathrm{C}$. The isolated cells were washed twice in ice-cold CMF PBS and blocked with 2.4G2 anti-Fc $\gamma$ RIII/II in ice-cold staining buffer (CMF PBS $+5 \%$ FBS) for $10 \mathrm{~min}$ on ice. Following washing with ice-cold staining buffer, the cells were stained with antibody staining cocktail (CD11c and MHC class II) for 20 min on ice in the dark. Finally, the cells were washed with ice-cold staining buffer two times and resuspended in $400 \mu \mathrm{l}$ of ice-cold staining buffer for FACS analysis.

\subsection{Statistical analysis}

All results are expressed as mean \pm standard deviation (SD). Statistical significance was assessed using t-test and a one-way analysis of variance (ANOVA) and post hoc Tukey multiple comparison test. All statistical analysis was performed using GraphPad PRISM software (GraphPad 
Software, Inc.) All statistical significance is denoted by $* p<0.05, * * p<0.01$, and $* * * p<0.001$.

\section{Results and discussion}

\subsection{Preparation and characterization of T-CAP}

T-CAP was prepared by conjugation with L-cysteine hydrochloride by DCC/NHS activated coupling reaction

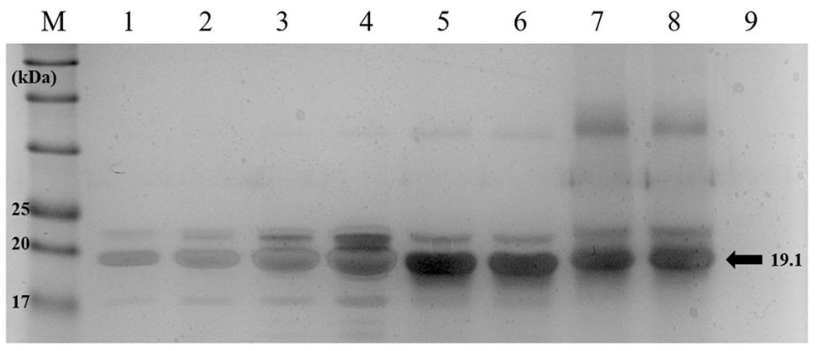

Fig. 4 Evaluation of protein structure of M5BT released from M5BT/CAP MPs and M5BT/T-CAP MPs by SDS-PAGE. Lane M: protein marker; Lane 1-4: native M5BT $(2.5-20 \mu \mathrm{g})$; Lane 5-6: M5BT released from CAP MPs; Lane 7-8: M5BT released from T-CAP MPs and Lane 9: protein-unloaded T-CAP MPs under nitrogenous condition to prevent the oxidation of sulfhydryl group. The reaction scheme for synthesis of T-CAP is shown in Fig. 2. The coupling of cysteine and CAP was confirmed by proton nuclear magnetic resonance $\left({ }^{1} \mathrm{H}-\mathrm{NMR}\right)$ as shown in Fig. 3. The peaks from acetate and thiol protons appeared in the H-NMR spectrum of T-CAP, indication of successful conjugation of cysteine in T-CAP. The thiol content in T-CAP was $16.04 \mu \mathrm{mol} / \mathrm{g}$ polymer as determined by Ellman's method although we controlled the thiol content in the T-CAP because too much contents of thiol group in the T-CAP induce disulfide bonds among thiol groups in the T-CAP.

\subsection{Preparation of T-CAP MPs}

\subsubsection{Characterization of M5BT/T-CAP MPs}

The loading content and encapsulation efficiencies of M5BT in the T-CAP MPs is shown in Table 1. The loading content of M5BT/CAP MPs and M5BT/T-CAP MPs were $4.62 \%(\mathrm{w} / \mathrm{w})$ and $4.97 \%(\mathrm{w} / \mathrm{w})$, respectively, showing similar antigen amount per MPs. And encapsulation efficiency of M5BT/CAP MPs and M5BT/T-CAP MPs were
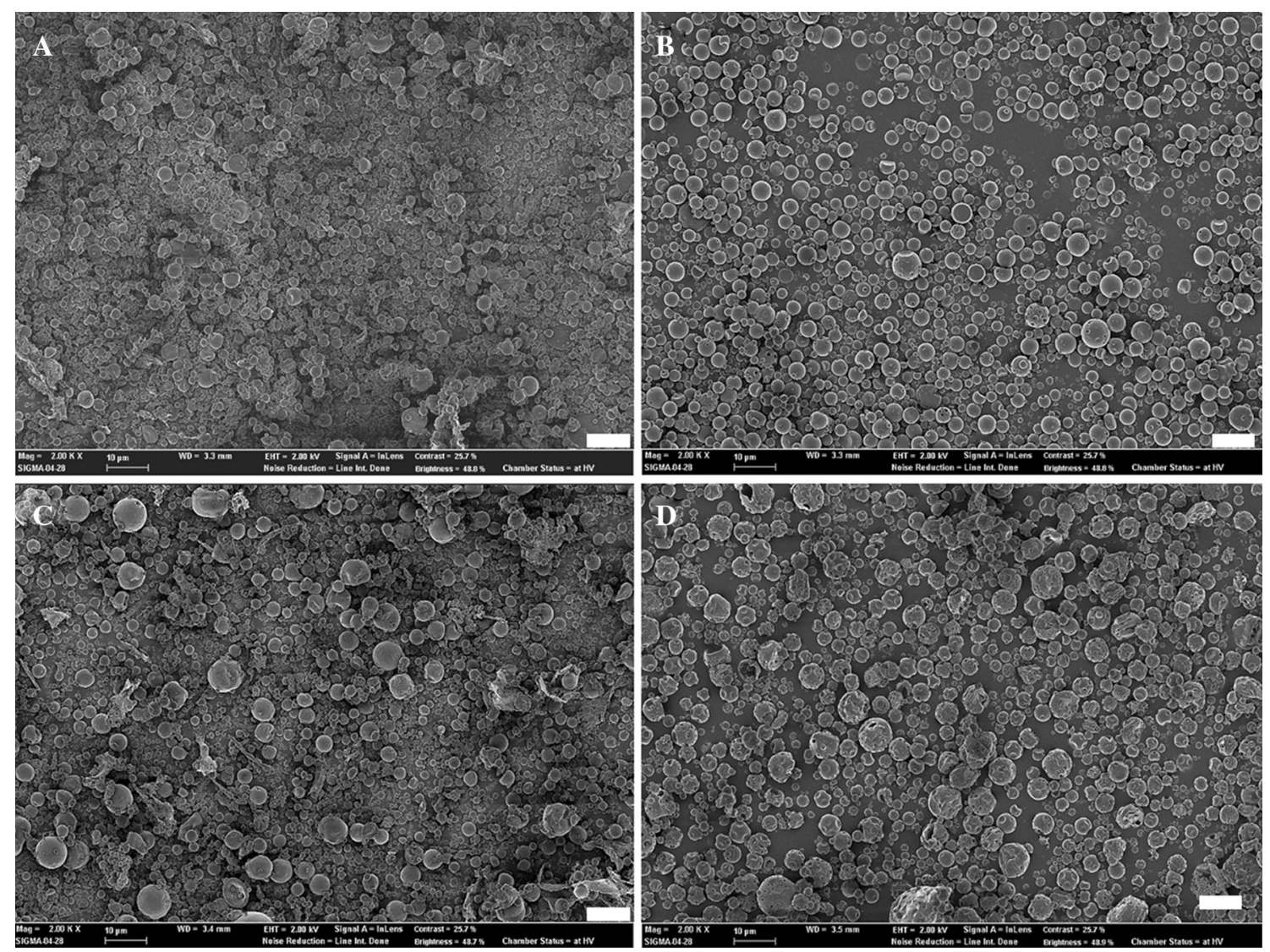

Fig. 5 Analysis of morphology of MPs by FE-SEM. A CAP MPs, B T-CAP MPs, C M5BT/CAP MPs and D M5BT/T-CAP MPs [Magnification $2000 \times$ and scale bar (white bar): $10 \mu \mathrm{m}]$ 

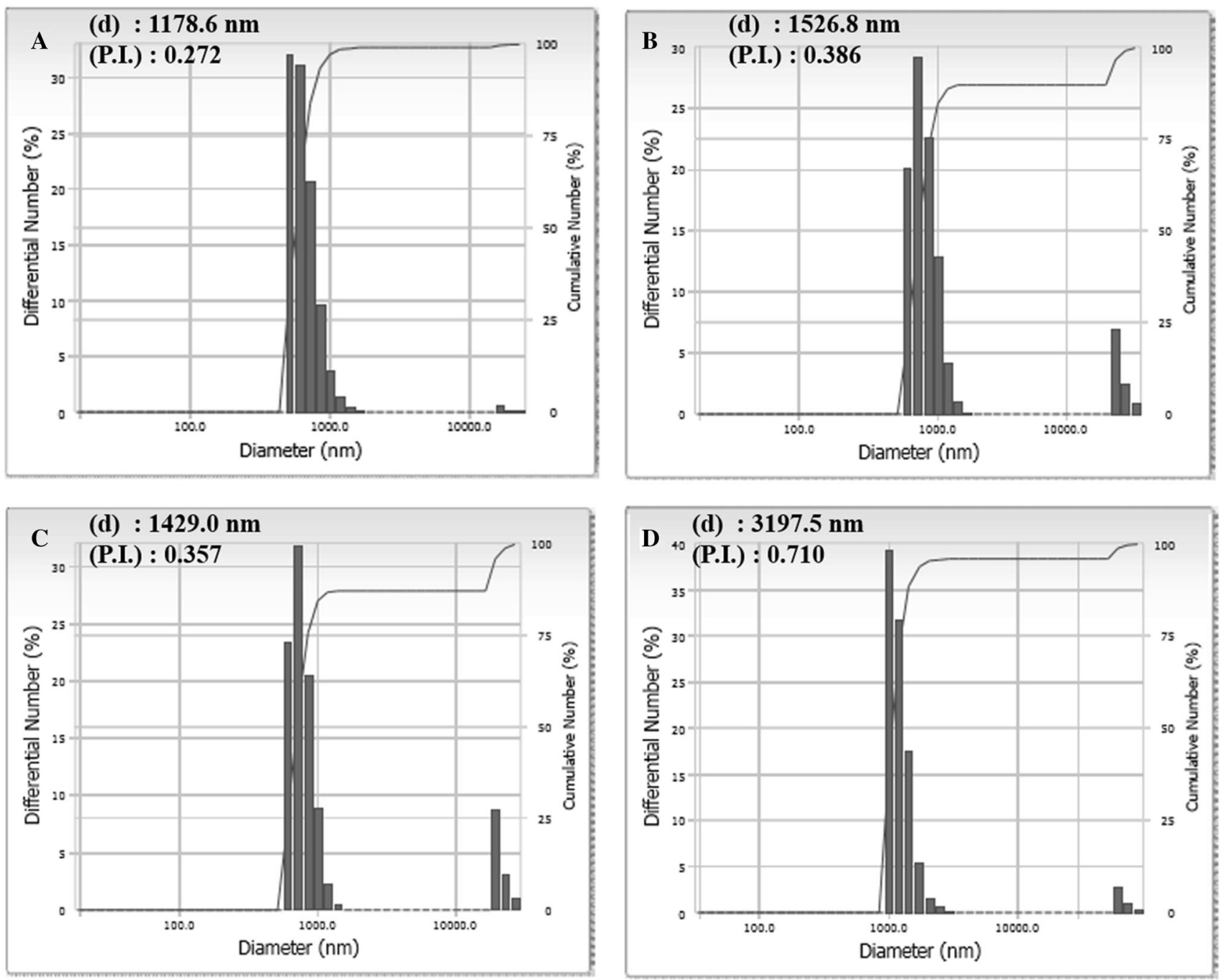

Fig. 6 Measurement of dynamic light scattering for size of MPs. A CAP MPs, B T-CAP MPs, C M5BT/CAP MPs and D M5BT/T-CAP MPs. (d) means average diameter of MPs and (P.I.) is polydispersity index

$82.2 \%(\mathrm{w} / \mathrm{w})$ and $72.1 \%(\mathrm{w} / \mathrm{w})$, respectively, suggesting that different solvent used in MP preparation may affect the encapsulation efficiency between them.

During the loading of vaccine into the MPs, it is possible to occur degradation of recombinant proteins. Therefore, the structural integrity of the M5BT released from M5BT/ T-CAP MPs was evaluated by SDS-PAGE as shown in Fig. 4. It was found that there was no difference of molecule weight of M5BT among native M5BT and, released M5BT from CAP and T-CAP, suggestion of retaining of structural integrity of the M5BT during encapsulation of M5BT into MPs although secondary structure of the released M5BT should be checked by the circular dichrorism spectrometer to identify stability of epitopes recognized by $\mathrm{B}$ cell receptors (BCRs) and $\mathrm{T}$ cell receptors (TCRs).

\subsubsection{Morphology and size of M5BT/CAP and M5BT/T- CAP MPs}

The morphologies of M5BT/CAP and M5BT/T-CAP MPs were observed by FE-SEM. Both MPs had well-formed spherical particles with smooth surfaces as shown in Fig. 5. Also, the sizes of MPs were analyzed by DLS as shown in Fig. 6. The average sizes of CAP and T-CAP MPs were 1.18 and $1.53 \mu \mathrm{m}$ respectively and them of M5BT/CAP and M5BT/T-CAP MPs were 1.43 and $3.20 \mu \mathrm{m}$ respectively, indicating that the sizes of M5BT-loaded MPs were increased after loading of M5BT into the MPs. It was reported that MPs smaller than $10 \mu \mathrm{m}$ can be efficiently taken up by Microfold cells ( $\mathrm{M}$ cells, antigen uptaking cells) of Peyer's patches in ileum [14]. Moreover, MPs with this range in diameter can be internalized through 


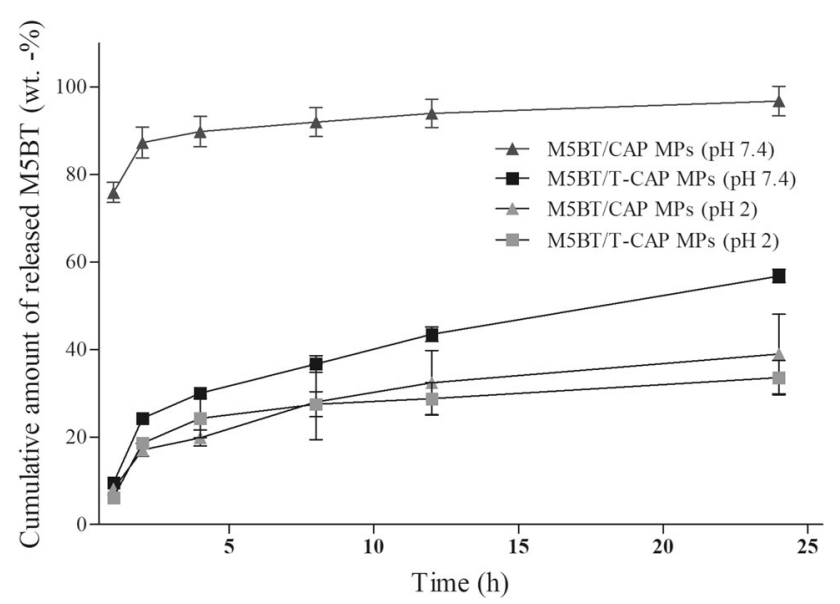

Fig. 7 In vitro release profile of M5BT protein from M5BT/CAP and M5BT/T-CAP MPs at simulated gastrointestinal $\mathrm{pH}$ MPs $(10 \mathrm{mg} / \mathrm{ml})$ were suspended in different $\mathrm{pH}$ buffer ( $\mathrm{pH} 2$ and $\mathrm{pH}$ 7.4). Protein concentration was measured by micro BCA assay. All values represent the mean $\pm \mathrm{SD}(\mathrm{n}=3)$

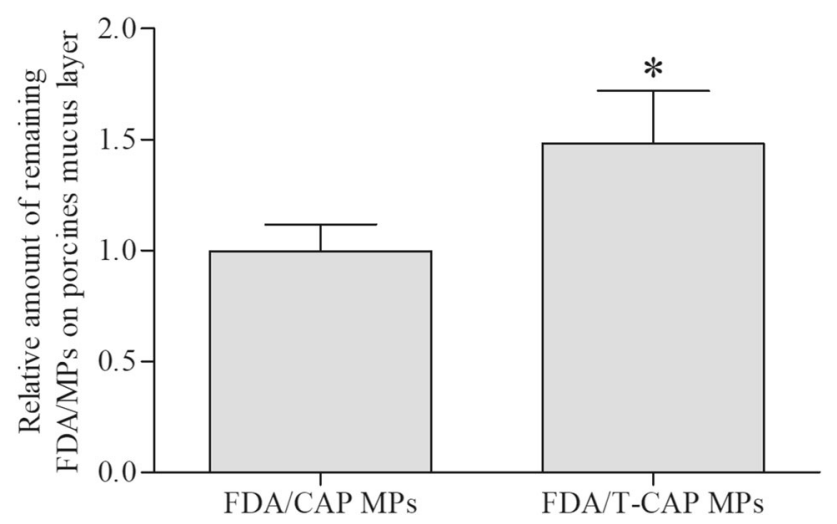

Fig. 8 Analysis of mucoadhesive property of MPs in small intestine. $4 \mathrm{mg}$ of each of FDA-loaded MPs was dispersed on a freshly excised porcine intestinal mucosa and incubated at $37{ }^{\circ} \mathrm{C}$ for $1 \mathrm{~h}$ with shaking at $100 \mathrm{rpm}$. The MPs attached on the mucosa were collected and remaining concentration of each MPs was calculated by measuring the absorbance of FDA at $490 \mathrm{~nm}(\mathrm{n}=3)(* p<0.05$ by t-test $)$

phagocytosis by APCs playing a crucial role in initiating innate immune response [21].

\subsubsection{In vitro release behavior of M5BT from CAP and T- CAP MPs}

The in vitro release profile of M5BT from CAP and T-CAP MPs was investigated at simulated gastric acid ( $\mathrm{pH} 2)$ and simulated intestinal fluid conditions ( $\mathrm{pH} \mathrm{7.4)}$ for $24 \mathrm{~h}$ and was shown in Fig. 7. The release profiles of M5BT from MPs were presented as the percentage of amount of M5BT released from MPs with respect to the amount of M5BT loaded in MPs. The results indicated that the release of M5BT from M5BT/T-CAP MPs was higher at $\mathrm{pH} 7.4$ compared to $\mathrm{pH} 2$ due to the $\mathrm{pH}$-sensitivity of $\mathrm{CAP}$ by the carboxylic group of phthalic acid in the CAP, as expected. Thiolation of CAP also exhibited gastro-resistant property of CAP at simulated gastric solution $(\mathrm{pH} 2)$ with releasing $18.6 \pm 1.18$ and $17.1 \pm 1.42 \%$ from MPs at $2 \mathrm{~h}$, respectively. The burst release effect of M5BT/CAP MPs at $\mathrm{pH}$ 7.4 was obtained within $2 \mathrm{~h}$ due to the rapid dissolution of CAP above $\mathrm{pH}$ 6.2. M5BT release profile of M5BT/T-CAP MPs at simulated ileum $\mathrm{pH}(\mathrm{pH}$ 7.4) showed $43.5 \pm 1.63$, $56.8 \pm 1.49 \%$ at 12 and $24 \mathrm{~h}$ respectively whereas release of M5BT from T-CAP MPs was $28.8 \pm 3.68$, $33.6 \pm 3.92 \%$ at 12 and $24 \mathrm{~h}$ respectively at $\mathrm{pH} 2$. The release behavior of M5BT from both MPs at $\mathrm{pH} 2$ might be resulted from the diffusion of M5BT protein inside/outside the MPs. Interestingly, water solubility of the CAP above pH 6.4 was changed into above around $\mathrm{pH} 7.4$ after thiolation of CAP, which is very similar with thiolated hydroxyl propyl methyl cellulose phthalate (T-HPMCP) reported in a previously study [13]. Therefore, it is suggested that M5BT was rapidly released from M5BT/CAP MPs at pH 7.4 through burst-out mechanism because the CAP MPs are completely water-soluble at that $\mathrm{pH}$ whereas M5BT was slowly released from M5BT/T-CAP MPs at $\mathrm{pH}$ 7.4 through diffusion mechanism because the T-CAP MPs are not water-soluble at that $\mathrm{pH}$.

Eldridge et al., reported that total number of microparticles within Peyer's patches of intestine increased until day 4 and microparticles less than $<5 \mu$ m were specifically taken up into the Peyer's patches of the GALT [14]. The results imply that M5BT/T-CAP MPs can continuously release antigen from MPs after M5BT/T-CAP MPs were internalized into Peyer's patches.

\subsubsection{Mucoadhesive property of T-CAP MPS}

Mucoadhesive property of T-CAP MPs was evaluated by ex vivo experiment using freshly excised porcine intestinal mucosa with FDA-loaded MPs as a fluorescence marker. The amount of FDA-loaded MPs attached on freshly excised porcine intestine at $37{ }^{\circ} \mathrm{C}$ is shown in Fig. 8. The results indicated that the mucoadhesion of T-CAP MPs was 1.48-fold higher than that of CAP MPs after $1 \mathrm{~h}$ of incubation. Due to the enhanced mucoadhesion of T-CAP MPs, it is possible that M5BT/T-CAP MPs continuously release M5BT from T-CAP MPs remaining on the mucus layer.

\subsection{In vivo immunization in murine model}

\subsubsection{Flow cytometric detection of MHC class II- expressing cells in Peyer's patches in ileum}

Immune cells located throughout the intestinal lamina propria, especially in Peyer's patches, play a crucial role in sampling and processing luminal antigen for presentation 
A
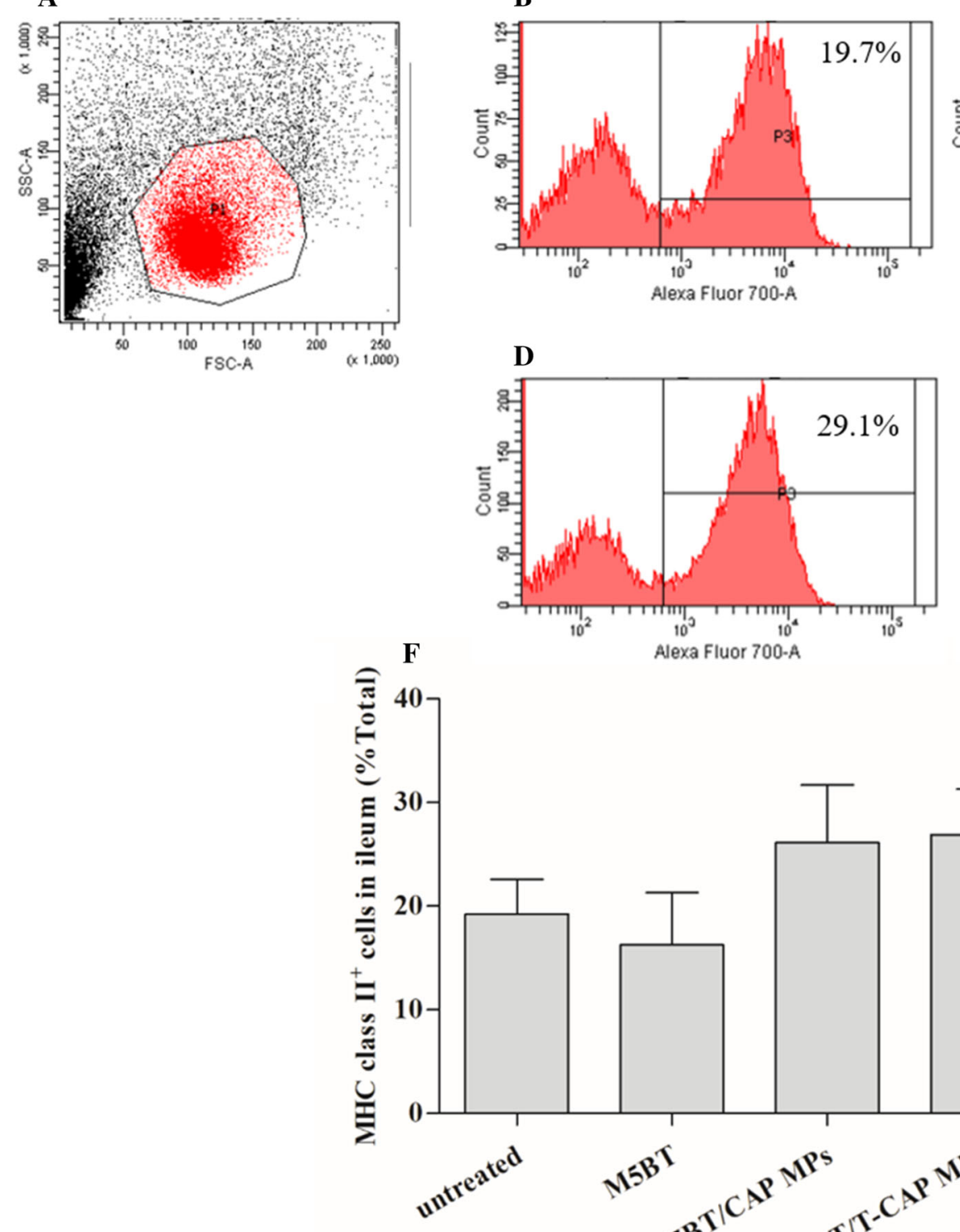

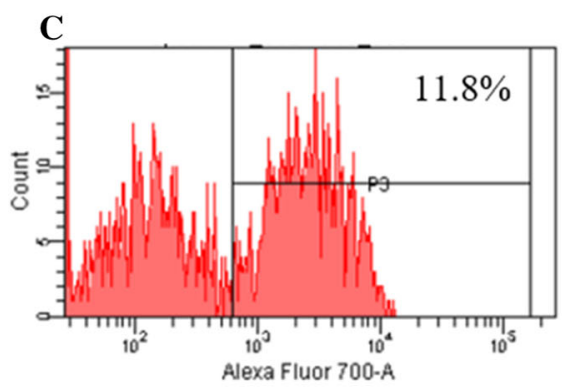

$\mathbf{E}$

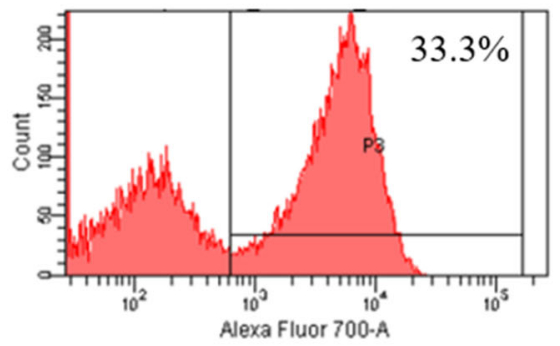

Fig. 9 Flow cytometric detection of MHC class II-expressing cells in Peyer's patches from immunized mice. Peyer's patches were collected from the mice immunized with M5BT/CAP or M5BT/TCAP MPs. Isolated cells were stained with MHC class II markers prior to detection by FACS. The percentage of MHC class II-positive cells is indicated. A; gating area and $\mathbf{B}-\mathbf{F}$ MHC class II-positive cells is indicated. B; untreated, C; M5BT, D; M5BT/CAP MPs, E; M5BT/ T-CAP MPs and F; the mean percentage of MHC class II-positive cells from total population (\%total) $(\mathrm{n}=3)$. SSC-A (side scatter) means granularity and FSC-A (forware scatter) means cell size to B and T cells [22]. To determine the population of APCs interacting with the antigen to initiate adaptive immune response in vivo, immune cells from Peyer's patches in ileum were isolated and analyzed by flow cytometry. Here, APCs populations in Peyer's patches were analyzed using the MHC class II surface marker as shown in Fig. 9. After gating, major immune cell populations expressing MHC class II were identified. The average of major immune cell population expressing MHC class II of the mice fed with
M5BT via MPs (M5BT/CAP MPs: $26.1 \%$ and M5BT/TCAP MPs: $26.87 \%$ ) was increased when compared to that of mice fed with M5BT only (16.23\%) although there is not much difference of MHC class II-positive cells between CAP and T-CAP MPs as shown in Fig. 9F.

In addition, CD11c-positive cells in Peyer's patches from immunized mice with M5BT/T-CAP MPs were increased in total populations (untreated, M5BT only: 0.30\%, M5BT/CAP MP: $0.47 \%$ and M5BT/T-CAP MPs: 


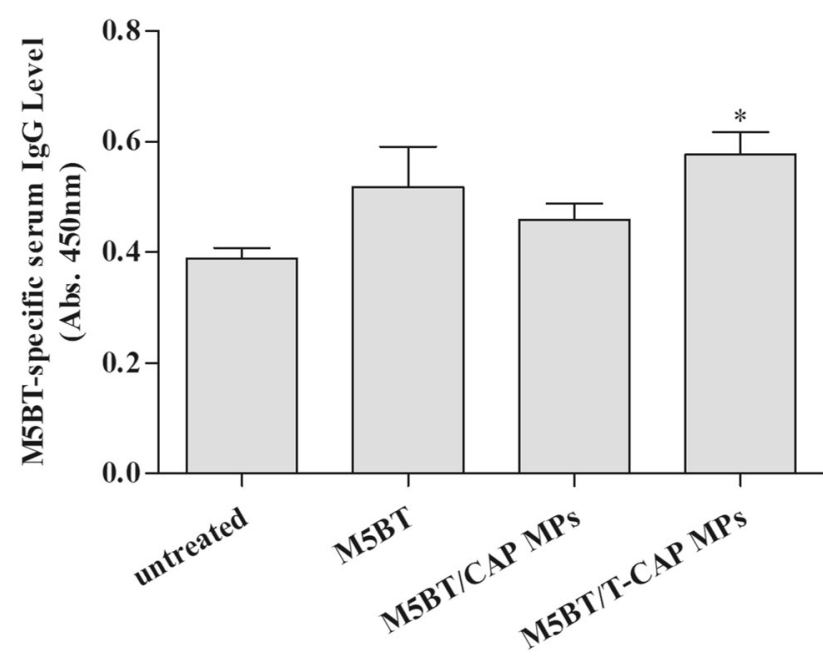

Fig. 10 M5BT-specific immune response after oral immunization with MPs. Anti-M5BT serum IgG levels at 4 weeks after immunization were measuring using ELISA. All values represents the mean $\pm \mathrm{SD}(\mathrm{n}=5)(* p<0.05$, one-way ANOVA $)$

$0.6 \%$ ). Increased population of MHC class II-expressing cells such as APCs in Peyer's patches may influence the production of IgA in intestine.

\subsubsection{M5BT-specific antibody production after oral immunization with MPs}

To evaluate the immune-enhancing effect of M5BT-loaded MPs by oral route, mice were immunized with M5BT alone, M5BT/CAP MPs and M5BT/T-CAP MPs by oral gavage.

Antigen-specific immunoglobulin in serum and fecal samples from immunized with samples were analyzed by M5BT-specific ELISA. M5BT-specific ELISA was conducted by coating immunoplate with M5BT recombinant antigen.

To assess the systemic immune response after oral immunization with samples, anti-M5BT IgG levels in serum samples from immunized mice were analyzed by ELISA. Among the immunized groups, mice immunized with M5BT/T-CAP MPs showed the highest M5BTspecific IgG level compared to untreated, M5BT only, and M5BT/CAP MPs groups as shown in Fig. 10.

In addition, anti-M5BT IgA in fecal sample from immunized mice with M5BT-loaded MPs was significantly higher than that of mice immunized with M5BT itself without carrier as shown in Fig. 11. The results can be explained that polymeric carrier delivers antigen to lymphoid tissue to induce antigen-specific immune response. Interestingly, M5BT/T-CAP MPs exhibited more IgA production compared to M5BT/CAP MPs at 2 and 4 weeks due to longer transit time of antigens by the mucoadhesive MPs although difference of feces IgA between 2 and 4 weeks resulted in the different final concentration of fecal pellets affecting lower absorbance of fecal samples at 4 weeks. After normalization of each IgA levels by IgA levels of control groups to compensate the final fecal concentration, M5BT-specific IgA levels were significantly higher at 4 weeks in mice immunized with M5BT/T-CAP MPs as shown in Fig. 11C.

In conclusion, $\mathrm{pH}$-sensitive and mucoadhesive T-CAP was prepared and characterized for delivering FMD subunit vaccine into small intestine because oral vaccination of protein antigen has limitation due to its poor immunogenicity and low bioavailability despite of many advantages compared to parenteral administration. It activated more producing $\operatorname{Ig} \mathrm{A}$, the most important immunoglobulin for preventing FMD at the early stage of infection, as a mucosal oral vaccine adjuvant. It was also found that T-CAP MPs resulted in the elevated population of MHC class $\mathrm{II}^{+}$cells in Peyer's patches because antigen uptaken by M-cells from lumen can be uptaken and be transported to lymph node by immune cells (DCs, macrophages and B cells) to active both innate immunity and adaptive immunity. This indicated that oral immunization of mucosal subunit vaccine via T-CAP MPs effectively delivered the

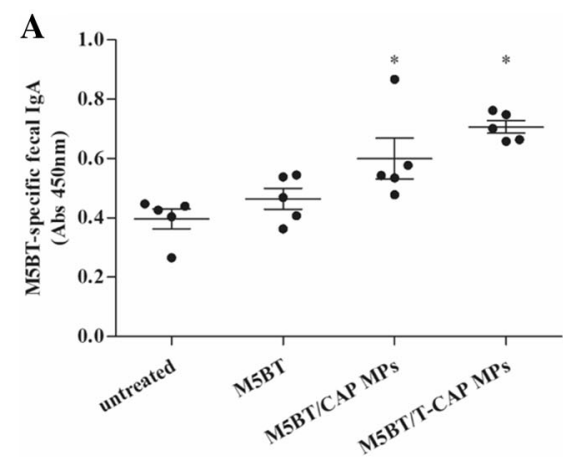

Fig. 11 Anti-M5BT IgA level in feces after oral immunization with MPs. Fecal samples were taken from mice at A 2 weeks, B 4 weeks and $\mathbf{C}$ relative anti-M5BT IgA level compared with untreated group.
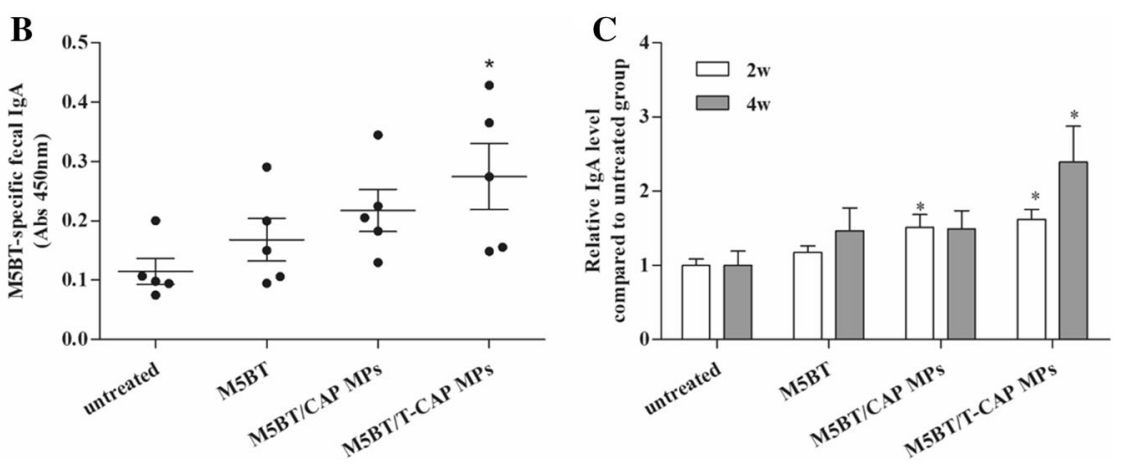

Antibody levels were analyzed by ELISA ( $\mathrm{n}=5$, error bars represent standard deviations; $* p<0.05$, one-way ANOVA) 
vaccine to Peyer's patches eliciting mucosal IgA response. It will make a step forward into a promising oral subunit vaccine development in livestock industry.

Acknowledgements This work was supported by the Ministry of Science, ICT and Future Planning (Project No. 2016R1D1A1B03933491). This work was also supported by the Technological Innovation R\&D Program (S2273990) funded by the Small and Medium Business Administration(SMBA, Korea). The first authors were supported by Brain Korea 21 plus program.

\section{Compliance with ethical standards}

Conflicts of interest The authors have no financial conflicts of interest.

Ethical statement Mouse in vivo experiment were carried out following the policy and regulations for the care and use of laboratory animal (Laboratory Animal Center, Seoul National University, Korea). All of the protocols were reviewed and approved by the Animal Care and Use Committee at Seoul National University (SNU150514-3-2).

\section{References}

1. Sobrino F, Sáiz M, Jiménez-Clavero MA, Núñez JI, Rosas MF, Baranowski E, et al. Foot-and-mouth disease virus: a long known virus, but a current threat. Vet Res. 2001;32:1-30.

2. Rodriguez LL, Grubman MJ. Foot and mouth disease virus vaccines. Vaccine. 2009;27:D90-4.

3. Purcell AW, McCluskey J, Rossjohn J. More than one reason to rethink the use of peptides in vaccine design. Nat Rev Drug Discov. 2007;6:404-14.

4. Domingo E, Mateu MG, Martínez MA, Dopazo J, Moya A, Sobrino F. Genetic variability and antigenic diversity of foot-andmouth disease virus. Virus variability, epidemiology and control. Berlin: Springer; 1990. p. 233-66.

5. Lee HB, Piao DC, Lee JY, Choi JY, Bok JD, Cho CS, et al. Artificially designed recombinant protein composed of multiple epitopes of foot-and-mouth disease virus as a vaccine candidate. Microb Cell Fact. 2017;16:33.

6. Brownlie J. Clinical aspects of the bovine virus diarrhoea/mucosal disease complex in cattle. In Pract. 1985;7:195-202.

7. Maloy KJ, Donachie AM, O'Hagan DT, Mowat AM. Induction of mucosal and systemic immune responses by immunization with ovalbumin entrapped in poly(lactide-co-glycolide) microparticles. Immunology. 1994;81:661-7.
8. Wang S, Liu H, Zhang X, Qian F. Intranasal and oral vaccination with protein-based antigens: advantages, challenges and formulation strategies. Protein Cell. 2015;6:480-503.

9. Cubillos C, de la Torre BG, Jakab A, Clementi G, Borrás E, Bárcena $\mathrm{J}$, et al. Enhanced mucosal immunoglobulin A response and solid protection against foot-and-mouth disease virus challenge induced by a novel dendrimeric peptide. J Virol. 2008;82:7223-30.

10. Hussan S, Santanu R, Verma P, Bhandari V. A review on recent advances of enteric coating. IOSR J Pharm. 2012;2:5-11.

11. Shimojo AAM, Galdames SEM, Perez AGM, Ito TH, Luzo ÂCM, Santana MHA. In Vitro Performance of Injectable Chitosan-Tripolyphosphate Scaffolds Combined with Platelet-Rich Plasma. Tissue Eng Regen Med. 2016;13:21-30.

12. Song WY, Liu GM, Li J, Luo YG. Bone Morphogenetic Protein-2 Sustained Delivery by Hydrogels with Microspheres Repairs Rabbit Mandibular Defects. Tissue Eng Regen Med. 2016;13:750-61.

13. Singh B, Maharjan S, Jiang T, Kang SK, Choi YJ, Cho CS. Attuning hydroxypropyl methylcellulose phthalate to oral delivery vehicle for effective and selective delivery of protein vaccine in ileum. Biomaterials. 2015;59:144-59.

14. Eldridge JH, Hammond CJ, Meulbroek JA, Staas JK, Gilley RM, Tice TR. Controlled vaccine release in the gut-associated lymphoid tissues. I. Orally administered biodegradable microspheres target the Peyer's patches. J Control Release. 1990;11:205-14.

15. Kim SH, Jang YS. Antigen targeting to $M$ cells for enhancing the efficacy of mucosal vaccines. Exp Mol Med. 2014;46:e85.

16. Leitner VM, Walker GF, Bernkop-Schnürch A. Thiolated polymers: evidence for the formation of disulphide bonds with mucus glycoproteins. Eur J Pharm Biopharm. 2003;56:207-14.

17. Bernkop-Schnürch A, Steininger S. Synthesis and characterisation of mucoadhesive thiolated polymers. Int $\mathrm{J}$ Pharm. 2000;194:239-47.

18. Singh B, Jiang T, Kim YK, Kang SK, Choi YJ, Cho CS. Release and cytokine production of $\mathrm{BmpB}$ from $\mathrm{BmpB}$-loaded $\mathrm{pH}$-sensitive and mucoadhesive thiolated eudragit microspheres. J Nanosci Nanotechnol. 2015;15:606-10.

19. Sander JS, Studart AR. Multiwalled functional colloidosomes made small and in large quantities via bulk emulsification. Soft Matter. 2014;10:60-8.

20. Geem D, Medina-Contreras O, Kim W, Huang CS, Denning TL. Isolation and characterization of dendritic cells and macrophages from the mouse intestine. J Vis Exp. 2012;21:e4040.

21. Foged C, Brodin B, Frokjaer S, Sundblad A. Particle size and surface charge affect particle uptake by human dendritic cells in an in vitro model. Int J Pharm. 2005;298:315-22.

22. Holmgren J, Czerkinsky C. Mucosal immunity and vaccines. Nat Med. 2005;11:S45-53. 